\title{
NOTICEABILITY AND RECALL OF VISUAL ELEMENTS ON PACKAGING
}

\author{
Dorotea Kovačević (D), Maja Brozović (i) \\ University of Zagreb, Faculty of Graphic Arts, Zagreb, Croatia
}

\begin{abstract}
Product packaging protects the content from the environment, but also serves to display information relevant to consumers. Some product information, like warning messages, should be both noticeable and memorable. The purpose of the study was to explore how people pay attention to visual elements on packaging and how well they recall them. The investigation was especially directed towards a particular visual element - a safety pictogram. The study consisted of two parts: an eye tracking experiment which measured the participants' visual attention while viewing the packaging, and a followup memory test which assessed the ability of the participants to recall elements from the packaging. The packaging was designed especially for the purpose of the experiment. It was presented on-screen and viewed by 130 participants. The visual elements displayed on the packaging were: an illustration, a product name, a logo, a pictogram and the quantity information. Eye-tracking measures used were time to first fixation on each of the visual elements on the packaging and total fixation duration on an element. A list test was used for assessing the recall of visual information in the absence of the packaging. The illustration was the visual element recalled by most of the participants (reported by $80 \%$ of the participants), followed by the product name (reported by 59\% of the participants). Only $18 \%$ of the participants mentioned that they saw the pictogram. The analysis of the eye movements and the visual attention showed that the most easily noticed visual element was the product name, while the illustration had the longest viewing period. Furthermore, the participants who detected the pictogram faster and viewed it longer also recalled it better. However, the association between visual attention and memory was not revealed for all visual elements on the packaging. The findings could be relevant for designers and those interested in the communication aspect of packaging.
\end{abstract}

Key words: packaging, eye-tracking, recall, pictogram

\section{INTRODUCTION}

The basic role of packaging is to protect its content from the environment. Concurrently, it serves to display information relevant to consumers. Some product information, like warning messages, should be both noticeable and memorable. Noticeability is necessary because if a warning message is not detected, it can have no effect on safe behavior (Young et al, 1999). The memorability of a warning is important because warning information might not be accessible during the use of a product and exposure to associated hazards (Wogalter et al, 2002). A high level of warning recall was found to be related to compliance behavior (Wogalter et al, 1991) and the perception of the safety of the product (Stark et al, 2008).

Previous studies have shown that memorability and visual attention are linked. This was demonstrated in the case of pictures (Mancas et al, 2013), advertisements (Crespo et al, 2007), and also in the case of warning labels (Strasser et al, 2012). Many studies that investigated the association between attention and memory employed eye tracking technology (Krugman et al, 1994; Strasser et al, 2012). For example, Krugman et al (1994) used an eye tracking measure (i.e. time spent attending to a warning) to demonstrate that longer viewing time can be associated with the viewer's performance at a recall test. The results of the study done by Strasser et al (2012) also indicated that directing attention to a warning can improve recall of the warning information. They measured the time to first fixation on the warning and the dwell time duration to investigate the connection between visual exploration and correct recall. The effectiveness of eye tracking evaluations in user-centered design research is well known. It was used as a tool for accessing how people view a stimuli and what attracts their attention in a great deal of studies in this field. To name a few, Velásquez (2013) used an eye tracker to investigate how users perceive different elements on a web page instead of using a survey. Renshaw et al (2003) also recorded eye movements of participants to explore how specific designs affect visual processing. Strasser et al (2012) demonstrated that eye movement measures can reflect viewing patterns of participants who observed textual and graphic warning labels.

In this study, our aim was to use eye tracking measures in the context of packaging design and users' information processing. The intention was to explore how people pay attention to different visual 
elements on packaging and how well they recall them. More particularly, we measured the amount of time the participants spent attending to each of the elements and tested its association with the participants' performance in a free memory recall test. The investigation was especially directed towards a particular visual element - a safety pictogram.

\section{METHODOLOGY}

The experiment consisted of two parts. The first part was an eye tracking measurement of the participants' visual attention while viewing the packaging. The second part was a follow-up memory test which assessed the ability of the participants to recall the visual elements on the packaging.

130 people (87 women and 43 men) were recruited, and all voluntarily participated in the study. Their age ranged from 19 to $40(M=22.31, S D=3.14)$. All had normal or corrected-to-normal vision.

The experiments were conducted in a laboratory testing room at the University of Zagreb, Faculty of Graphic Arts. The stimulus was a picture of packaging presented on-screen, on a Lenovo computer display (model LEN L1900pA) with a resolution set to $1280 \times 1024$ pixels and a refresh rate of $60 \mathrm{~Hz}$. The participants' visual attention was recorded with a Tobii Eye Tracker X60 (sampling rate of $60 \mathrm{~Hz}$, accuracy of 0.5 degree). Eye tracking measures used as dependent variables were: time to first fixation (in seconds) and total fixation duration (in seconds) on each element on the presented packaging. Figure 1 shows the packaging used as stimuli. The packaging was designed for a fictitious brand of air freshener. The main visual elements displayed on the packaging were: an illustration, a product name, a logo, a pictogram and the quantity information.

Before the experiment started, the participants were instructed that they will participate in eye tracking testing, and that their task will be to view the example of the packaging in the same way as they would in their everyday life. They were informed that the viewing time would be limited and that they would be given a question at the end of the testing. In order to avoid goal directed visual search, the participants were not informed about the type of the question. After calibration, the participants fixated a symbol " $x$ " in the middle of the screen and then the image of the packaging was presented in the duration of 5 seconds. Immediately after the packaging presentation, a list test was used for assessing the recall of visual information. The measurement of recall was done by asking the participants to list any elements of the packaging they could remember. After the completion of the task, the participants were debriefed and thanked.

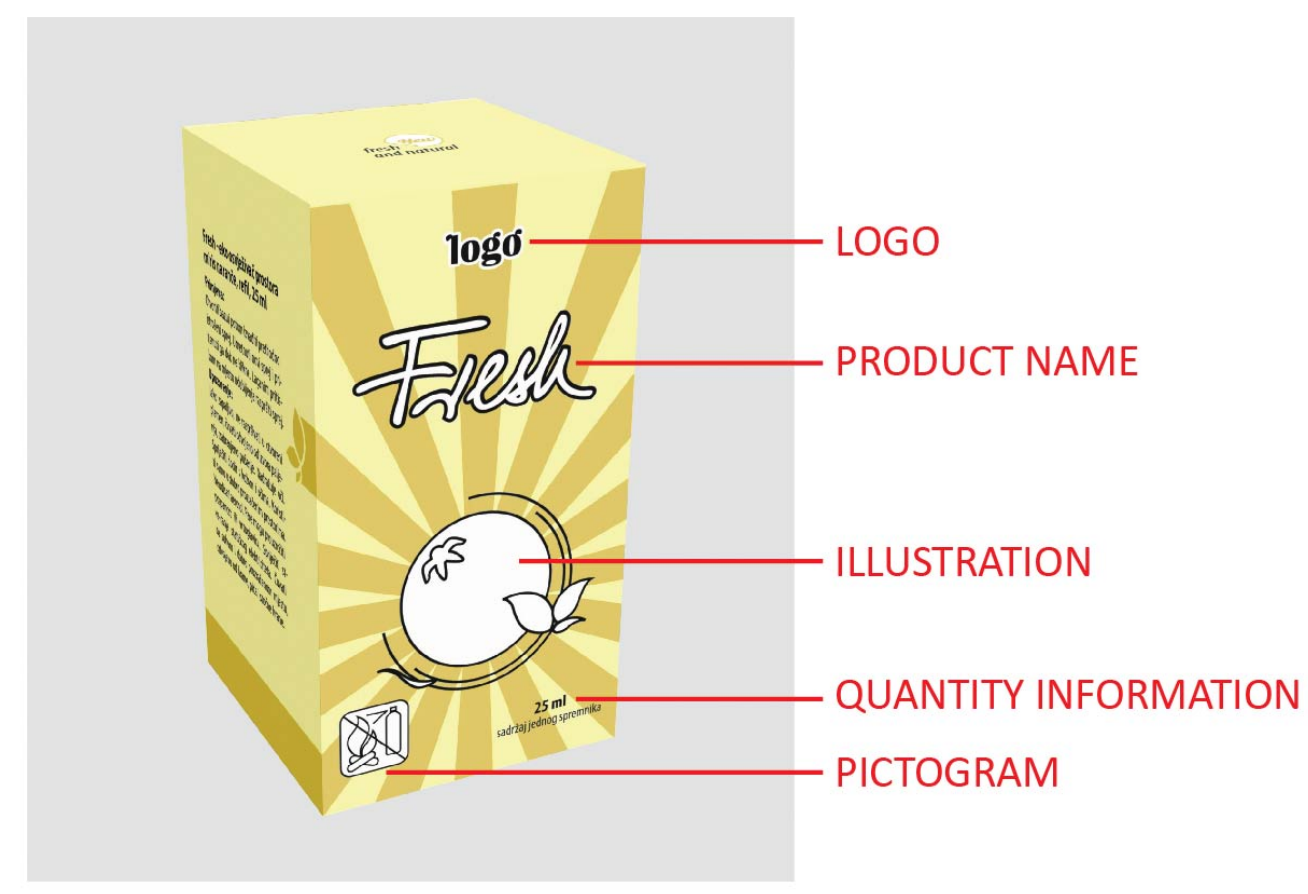

Figure 1: Picture of the packaging used as stimuli in the experiment 


\section{RESULTS}

\subsection{Visual attention}

Table 1 shows the descriptive statistics for the two measures of the participants' visual attention (i.e. time to first fixation and total fixation duration) grouped by visual elements. The time to first fixation and fixation duration data were analysed with a separate repeated-measures analyses of variance (ANOVA). The results indicated significant differences in the time to first fixation on different visual elements, $F(4,456)=245.42, p<0.01$. Since the pictogram was the focus of our investigation, pairwise t-tests were performed between the pictogram and each of the tested visual elements. The pictogram was fixated faster $(M=2.50, S D=0.56)$ than the quantity information $(M=2.78, S D=0.61), t(114)=-4.92, p<0.01$. However, it was fixated slower than the product name $(M=0.81, S D=0.43), t(114)=-23.60, p<0.01$, and the illustration $(M=1.02, S D=0.52), t(114)=-21.09, p<0.01$. There was no significant difference in the time to first fixation between the pictogram and the logo $(p=0.21)$.

A significant difference was found in the duration of fixations on different visual elements, $F(4,516)=$ $87.023, p<0.01$. The pairwise t-tests revealed that the pictogram was fixated longer $(M=2.83, S D=1.30)$ than the logo $(M=1.85, S D=1.63)$ and the quantity information $(M=2.42, S D=1.36)$. However, it was fixated less than the illustration $(M=4.22, S D=1.38)$ and the product name $(M=4.20, S D=1.14)$.

\subsection{Memory}

Figure 2 shows the results of the memory test. The bars represent the total number of participants who mentioned each of the visual elements in the test. Cochran's $Q$ test showed that the differences between memorizing the visual elements were significant, $\chi^{2}(4)=201.26, p<0.001$. The post hoc comparisons performed between the pictogram and each of the visual elements showed that the participants mentioned the pictogram less than the illustration $\chi^{2}=66.39, p<0.01$, and the product name, $\chi^{2}=37.04$, $p<0.01$. However, there was no significant difference in memorability between the pictogram and the logo $(p>0.05)$, and between the pictogram and the quantity information $(p>0.05)$.

Table 1: Descriptive statistics for eye tracking measurements

\begin{tabular}{|c|c|c|c|c|c|c|}
\hline & & \multicolumn{5}{|c|}{ Visual element } \\
\hline & & Illustration & Product name & Logo & Pictogram & $\begin{array}{c}\text { Quantity } \\
\text { information }\end{array}$ \\
\hline \multirow{2}{*}{$\begin{array}{l}\text { Time to first } \\
\text { fixation }\end{array}$} & Mean & 1.02 & 0.81 & 2.34 & 2.50 & 2.77 \\
\hline & Standard Deviation & 0.52 & 0.43 & 0.88 & 0.56 & 0.61 \\
\hline \multirow{2}{*}{$\begin{array}{l}\text { Total fixation } \\
\text { duration }\end{array}$} & Mean & 4.21 & 4.20 & 1.85 & 2.83 & 2.42 \\
\hline & Standard Deviation & 1.38 & 1.14 & 1.63 & 1.29 & 1.36 \\
\hline
\end{tabular}

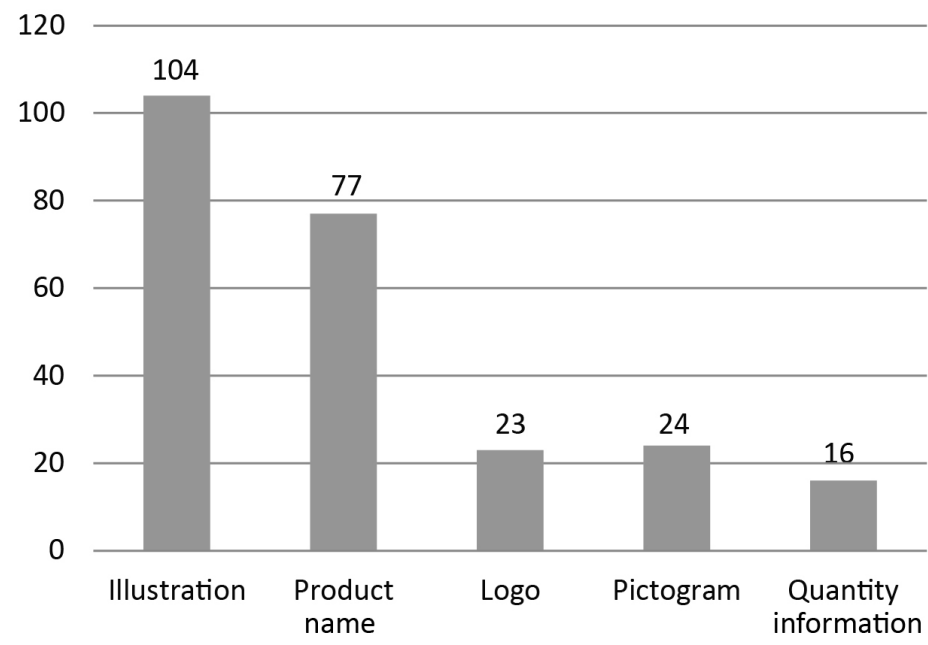

Figure 2: Number of participants who mentioned each of the visual elements in the memory test 


\subsection{Visual attention and memory}

The degree of association between the measures of visual attention and memory was calculated using Spearman's correlation analyses. Only the significant correlations are reported. There was a positive relationship between the time to first fixation on the logo and its memorability $r_{s}=.198, p<0.05$. There was a negative relationship between the time to first fixation on the pictogram and its memorability $r_{s}=-$ $.211, p<0.05$, indicating that the participants who noticed the pictogram faster also recalled it better. The analysis of the total fixation durations revealed a significant association between the duration of fixation on the pictogram and its memorability $r_{s}=.210, p<0.05$.

\section{DISCUSSION}

One of the intentions of the present study was to measure the visual attention that the participants direct towards elements on the packaging, with a special interest in the perception of the safety pictogram. Two measures were used; the time to first fixation on the visual element as a measure of noticeability (the ability of an element to attract attention) and the duration of fixations landing on the visual element as a measure of the ability of an element to hold one's attention. The results showed that the most noticeable object on the packaging was the product name, while the least noticeable object was the quantity information. The high level of noticeability could be the result of positioning the product name in the upper part of the packaging. The low noticeability of the quantity information can be explained not only by the lower positioning, but also by the low visual saliency derived from the small size of the element and the dominance of the background pattern.

Although the safety pictogram required less time to be noticed than the quantity information, it appears that it was not easily detected. This could be due to the design features of the pictogram. Previous research has shown that the pictogram's design characteristics such as size and thickness can affect its noticeability (Kovačević et al, 2018). In relation to the size of the packaging and the size of other elements, the dimensions of the pictogram were small, which probably had a negative influence on the pictogram's visual prominence and its ability to attract attention. On the other hand, the ability of the pictogram to hold one's attention was somewhat better. The results indicated that the participants viewed it longer than the quantity information, and also longer than the logo. This was expected, since previous research suggested that more complex visual forms need more fixations to be processed (Zusne, 1964). The structure of the pictogram used in our experiment was noticeably more complex than the logo.

The participants dedicated most of their attention to the illustration. This was the element on the packaging that had the longest total fixation duration. This result is in line with previous studies which found that graphics attracted more attention than other elements. For example, Piqueras-Fiszman et al (2013) investigated the consumers' attention to the design of jam jars and found that graphics presented on the packaging attracted more attention to itself than the text, resulting with increased fixation duration. In general, fixation duration tends to be longer on informative visual elements than on less informative elements (Unema et al, 2005). In our experiment, the illustration was the most informative object because it provided information about the most distinctive characteristic of the product - its fragrance. The longer fixation duration also indicates that the illustration was more engaging in some way (Just et al, 1976), or relevant for viewers (Jacob et al, 2003).

The results of the memory test supported the dominant role of the illustration, indicating that the illustration was the visual element recalled by most of the participants (reported by $80 \%$ of the participants), followed by the product name (reported by $77 \%$ of the participants). Only $18 \%$ of the participants mentioned that they saw the pictogram. The power of the illustration to be recalled better than the pictogram element can be explained by the visual saliency of the illustration. Some previously done memory-based studies have shown that the saliency of an object can be a good predictor of its memorability (Dubey et al, 2015; Borkin et al, 2016). The most prominent elements within an image are likely to be remembered (Dubey et al, 2015), and the illustration was undoubtedly the visual cue that stood out the most. It was designed as the largest and the brightest element on the packaging, with a central position highlighted by the background texture. Perhaps some other design features of the pictogram that make it more salient would generate better recall. Many warning researchers have reported about the connection between salient warnings and memorability. Frienmann (1988) found that the warning symbol which was recalled more often than the other symbols in the study had better 
graphic qualities which made it more conspicuous. Torres et al (2007) revealed that overtly positioned warnings in print ads provide a higher warning recall.

A higher recall of a warning message is often associated with deeper cognitive processing of the message, measured through a greater number of fixations and viewing duration (Crespo et al, 2007). Some of our results support this connection between recall and visual attention. The correlation analyses revealed that the participants who detected the pictogram faster and viewed it longer also recalled it better. Similar findings are reported by Strasser et al (2012) who found that warnings that attracted attention more quickly and had longer dwell time duration were associated with better recall. However, the association between visual attention and memory was not revealed for all visual elements in our study. The lack of consistency in results for all the elements is probably caused by the variety of their attributes that produced different visual effects. For example, the color and recognisability of an object enhance memorability (Borkin et al, 2013), but do not necessarily affect the measures of the visual attention. Also, participants tend to remember the warning messages which they perceive as being important (Lehto et al, 1993), so the perception of the relevancy of each of the elements on the packaging might also affected the recall test performance. Finally, increasing the amount of information on packaging may affect the recall of an individual element (Scammon, 1977), so, when analysing the obtained results, the effect of visual clutter on the packaging should definitely be taken into consideration.

\section{CONCLUSIONS}

Since packaging often provides product-related safety information, which is especially important to be recalled, a particular research focus in the present study was on the safety pictogram. By connecting the results of the free memory recall test with the eye tracking results, the study demonstrated the connection between attention and recall to some extent, but also touched upon some issues associated with safe product use.

The main finding of the study is that the memorability of the pictorial warning could benefit from emphasizing its noticeability. However, eye tracking results suggested that the link between the visual attention and the recall was not revealed for all elements on the packaging. Thus, any further conclusions about memorability and attention in reference to the design of the product packaging should not be generalized. Another finding is related to the communication role of the packaging. While the illustration and the product name had high levels of recall, the safety pictogram was recalled by only a small number of participants. This brings to light the questionable effectiveness of safety communication derived from the packaging design used in our study, and suggests that more effort should be put into testing the determinants of effective design of the safety pictorial in future research. Also, future studies should investigate the influence of color, which was, due to the preliminary nature of our study, omitted as an independent factor in the present work. In addition, various packaging designs and compositions of the elements could be differently perceived, so these variables should also be worthy of more research attention in the future.

\section{REFERENCES}

[1] Borkin, M. A., Bylinskii, Z., Kim, N. K., Bainbridge, C. M., Yeh, C. S., Borkin, D., Pfister, H., Oliva, A.: "Beyond memorability: Visualization recognition and recall", IEEE Transactions on Visualization and Computer Graphics 22(1), 519-528, 2016. doi: 10.1109/TVCG.2015.2467732.

[2] Borkin, M. A., Vo, A. A., Bylinskii, Z., Isola, P., Sunkavalli, S., Oliva, A., Pfister, H.: "What makes a visualization memorable?", IEEE Transactions on Visualization and Computer Graphics 19(12), 23062315, 2013. doi: 10.1109/TVCG.2013.234.

[3] Crespo, A., Cabestrero, R., Grzib, G., Quirós, P.: "Visual attention to health warnings in tobacco advertisements: An eye-tracking research between smokers and non-smokers", Studia Psychologica 49(1), 39-51, 2007.

[4] Dubey, R., Peterson, J., Khosla, A., Yang, M-H., Ghanem, B., :What makes an object memorable?", Proceedings of The IEEE International Conference on Computer Vision 2015, (ICCV, Santiago, Chile 2015), pages 1089-1097. doi: 10.1109/ICCV.2015.130.

[5] Frienmann, K., 1988.: "The effect of adding symbols to written warning labels on user behavior and receall", Human Factors: The Journal of the Human Factors and Ergonomics Society 30(4), 507-515, 1998. doi: 10.1177/001872088803000411. 
[6] Jacob, R.J.K., Karn, K.S.: "Commentary on Section 4 - Eye tracking in human-computer interaction and usability research: Ready to deliver promises", The Mind's Eye 573-605, 2003. doi: 10.1016/B978-044451020-4/50031-1.

[7] Just, M.A., Carpenter, P.A.: "Eye fixations and cognitive processes", Cognitive Psychology 8(4), pp.441-480, 1976. doi: 10.1016/0010-0285(76)90015-3.

[8] Kovačević, D., Brozović, M., Možina, K.: "Do prominent warnings make packaging less attractive?" Safety Science 110, 336-343, 2018. doi: 10.1016/j.ssci.2018.08.031.

[9] Krugman, D.M., Fox, R. J., Fletcher, J. E., Flscher, P. M., Rojas, T. H. : "Do adolescents attend to warnings in cigarette advertising? An eye-tracking approach", Journal of Advertising Research 34(6), 39-52, 1994.

[10] Lehto, M.R., Papastavrou, J.D.:"Models of the warning process: important implications towards effectiveness", Safety Science 16(5-6), 569-595, 1993. doi: 10.1016/0925-7535(93)90024-8.

[11] Mancas, M., Le Meur, O.: "Memorability of natural scenes: The role of attention", Proseedings of 2013 IEEE International Conference on Image Processing 2013, (ICIP, Melbourne, Australia, 2013), pages 196-200. doi: 10.1109/ICIP.2013.6738041.

[12] Piqueras-Fiszman, B. Velasco, B., Salgado-Montejo, A., Spence, C.: "Using combined eye tracking and word association in order to assess novel packaging solutions: A case study involving jam jars", Food Quality and Preference 28(1), 328-338, 2013. doi: 10.1016/j.foodqual.2012.10.006.

[13] Renshaw, J.A., Finalay, J.E., Tyfa, D., Ward, R.D.: "Designing for visual influence: An eye tracking study of the usability of graphical management information", Proceedings of Human-Computer Interaction - INTERACT 2003, (IFIP TC13, Zurich, 2003), pages 144-151, 2003.

[14] Scammon, D.L.: "'Information Load" and consumers", Journal of Consumer Research 4(3), 148, 1997. doi:10.1086/208690.

[15] Stark, E., Kim, A., Miller, C., Borgida, E.: "Effects of including a graphic warning label in advertisements for reduced-exposure products: Implications for persuasion and policy", Journal of Applied Social Psychology 38(2), 281-293, 2008. doi: 10.1111/j.1559-1816.2007.00305.x.

[16] Strasser, A.A., Tang, K.Z., Romer, D., Jepson C., Cappella J.N.: "Graphic warning labels in cigarette advertisements: Recall and viewing patterns", American Journal of Preventive Medicine 43(1), 4147, 2012. doi:10.1016/j.amepre.2012.02.026.

[17] Torres, I.M., Sierra, J.J., Heiser, R.S. "The effects of warning-label placement in print ads: A social contract perspective", Journal of Advertising 36(2), 49-62, 2007. doi:10.2753/JOA00913367360203.

[18] Unema, P.J.A., Pannasch, S., Joos, M., Velichkovsky, B. M.: "Time course of information processing during scene perception: The relationship between saccade amplitude and fixation duration", Visual Cognition 12(3), 473-494, 2005. doi: 10.1080/13506280444000409.

[19] Velásquez, J.D.: "Combining eye-tracking technologies with web usage mining for identifying Website Keyobjects", Engineering Applications of Artificial Intelligence 26(5-6), 1469-1478, 2003. doi: 10.1016/j.engappai.2013.01.003.

[20] Wogalter, M.S., Rashid, R., Clarke, S.W., Kalsher, M.J. :"Evaluating the behavioral effectiveness of a multi-modal voice warning sign in a visually cluttered environment", Proceedings of the Human Factors Society Annual Meeting 35(10), 718-722, 1991. doi:10.1177/154193129103501033.

[21] Wogalter, M.S., Conzola, V.C., Smith-Jackson, T.L.:"Research-based guidelines for warning design and evaluation", Applied Ergonomics 33(3), 219-230, 2002. doi: 10.1016/S0003-6870(02)00009-1.

[22] Young, S.L., Lovvoll, D.R. "Intermediate processing stages: Methodological condiserations for research on warnings", (Taylor \& Francis, London, 1999), pages 27-52.

[23] Zusne, L., Michels, K.M.: "Nonrepresentational shapes and eye movements", Perceptual and Motor Skills 18(1), 11-20, 1964. doi:10.2466/pms.1964.18.1.11.

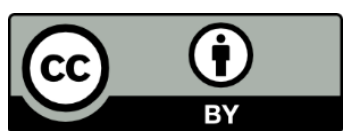

(C) 2018 Authors. Published by the University of Novi Sad, Faculty of Technical Sciences, Department of Graphic Engineering and Design. This article is an open access article distributed under the terms and conditions of the Creative Commons Attribution license 3.0 Serbia (http://creativecommons.org/licenses/by/3.0/rs/). 